\title{
Serum uric acid as a marker of microvascular damage in systemic sclerosis patients
}

\author{
Antonietta Gigante a , Biagio Barbano a , Giuseppe Barilaro a, Silvia Quarta a , Maria Ludovica Gasperini a , \\ Francesca Di Mario ${ }^{\mathrm{b}}$, Antonella Romaniello ${ }^{\mathrm{C}}$, Antonio Amoroso ${ }^{\mathrm{a}}$, Rosario Cianci ${ }^{\mathrm{a}}$, Edoardo Rosato ${ }^{\mathrm{a}, *}$ \\ a Department of Clinical Medicine, Clinical Immunology Unit-Scleroderma Center, Sapienza University of Rome, Italy \\ b Department of Nephrology and Dialysis Unit, Sapienza University of Rome, Italy \\ c Department of Clinical and Molecular Medicine, Cardiology Unit, S'Andrea Hospital, Sapienza University of Rome, Italy
}

\section{A R T I C L E I N F O}

\section{Article history:}

Received 2 November 2015

Revised 10 March 2016

Accepted 17 March 2016

Available online 18 March 2016

\section{Keywords:}

Uric acid

Systemic sclerosis

Renal Doppler ultrasound

Nailfold videocapillaroscopy

Systolic pulmonary artery pressure

microvascular damage

Endothelial dysfunction

\begin{abstract}
A B S T R A C T
Background: Microvascular damage of skin and internal organs is a hallmark of systemic sclerosis (SSc). Serum uric acid (UA) represents a marker of inflammation and endothelial dysfunction. The aims of this study were to evaluate the correlation between serum UA and intrarenal arterial stiffness evaluated by Doppler ultrasound in SSc patients with normal renal function. We also evaluated the correlation between serum UA and other clinical variables of the disease.

Methods: Forty-five SSc patients underwent clinical assessment, Doppler ultrasound of intrarenal arteries with evaluation of resistive index (RI), pulsatile index (PI), and systolic/diastolic ratio (S/D), echocardiography with systolic pulmonary artery pressure (PAPs), baseline pulmonary function tests, and nailfold videocapillaroscopy (NVC). In all patients serum UA was measured.

Results: The serum UA showed a significant positive correlation with $\mathrm{sCr}(\mathrm{r}=0.33, \mathrm{p}<0.0001)$ and PAPs $(\mathrm{r}=0.38, \mathrm{p}<0.01)>$ and negative correlation with CKD-EPI $(\mathrm{r}=-0.35, \mathrm{p}<0.01)$. The mean value of serum UA increased with severity of NVC damage. Using this cut-off value of $4.7 \mathrm{mg} / \mathrm{dl}$, the mean value of Doppler indices of intrarenal stiffness is significantly different $(p<0.05)$ in SSc patients with low normal or high normal serum UA.

Conclusions: Serum UA concentration is higher in patients with high microvascular damage than in patients with low microvascular damage. These preliminary data must be confirmed in large prospective studies.
\end{abstract}

(C) 2016 Elsevier Inc. All rights reserved.

\section{Introduction}

Systemic sclerosis (SSc) is a connective tissue disease characterized by endothelial dysfunction and fibrosis of both skin and internal organs. Endothelial dysfunction, microvascular and macrovascular damage are the hallmarks of SSc (Matucci-Cerinic et al., 2013).

The most important renal complication in SSc is scleroderma renal crisis, but latent renal involvement is present such as isolated reduced glomerular filtration rate (GFR), reduced renal functional reserve, microalbuminuria and increased intrarenal arterial stiffness. A reduction of glomerular filtration rate (GFR) can be present in SSc patients with normal serum creatinine ( $\mathrm{sCr}$ ) (Shanmugam and Steen, 2012). In SSc patients, GFR can be evaluated by estimated GFR (eGFR). Gigante et al. found that Chronic Kidney Disease Epidemiology Collaboration (CKD-EPI) equation provides more accurate eGFR in comparison to 7-

\footnotetext{
* Corresponding author at: Sapienza University of Rome, Department of Clinical Medicine, Clinical Immunology Unit-Scleroderma Center, Italy.

E-mail address: edoardo.rosato@uniroma1.it (E. Rosato).
}

variable MDRD (Gigante et al., 2012). Increased arterial stiffness is known to be associated with sclerodermic kidney dysfunction. It is known that Doppler renal ultrasound is a useful and non-invasive diagnostic tool to evaluate intrarenal arterial stiffness. The Doppler indices of intrarenal arterial stiffness showed a negative correlation with GFR and they increase with the severity of microvascular damage of hands.

Elevated serum uric acid (UA) has been shown to be associated with impaired endothelium-mediated relaxation, vascular stiffness (Sowers, 2013) and a restrictive left ventricular filling pattern or diastolic dysfunction (Hayden and Tyagi, 2004). Recently, the DETECT study has been performed with the aim of identifying predictors of pulmonary arterial hypertension (PAH) in patients with SSc. Serum urate has not been described previously as being predictive of PAH, but was identified as such in this study where low values were associated with a low PAH risk (Coghlan et al., 2013).

The aims of this study were to evaluate the correlation between serum UA and intrarenal arterial stiffness evaluated by Doppler ultrasound in SSc patients with normal renal function. We also evaluated the correlation between serum UA and other clinical variables of the disease. 


\section{Materials and methods}

Forty-five patients ( 36 female and 9 male; mean age $45 \pm$ 13.8 years) fulfilling the American College of Rheumatology/European League criteria for classification and diagnosis of SSc were enrolled in this study (van den Hoogen et al., 2013). Seventeen patients had limited cutaneous SSc (lcSSc) and 28 presented diffuse cutaneous SSc (dcSSc) as defined by LeRoy et al. (1998). Table 1 shows the SSc patients' epidemiological and clinical features.

All SSc patients underwent treatment with calcium channel blockers (nifedipine $30 \mathrm{mg} / \mathrm{day}$ ). None of the patients were treated with immunosuppressive agents (e.g. cyclophosphamide or mycophenolate mofetil), steroid, angiotensin-converting enzyme inhibitors (ACE-I), angiotensin II receptor blockers, diuretics or xanthine oxidase inhibitors. Patients with elevated serum creatinine $(\mathrm{sCr})$, elevated blood urea, urinary tract infections, abnormal urinary sediment, glomerulonephritis, kidney stones, anti-phospholipid-associated nephropathy, diabetes, cardiovascular diseases such as hypertension, myocardial infarction, arrhythmias, heart failure, hyperlipidemia, coagulopathy, obesity, scleroderma renal crisis or smokers were excluded.

The subjects' written consent was obtained according to the Declaration of Helsinki and the study was approved by the ethics committee of Sapienza University (IRB approval 3377/2014).

\section{Laboratory parameters}

Laboratory investigations included sCr (normal range: $0.5-0.9 \mathrm{mg} / \mathrm{dl}$ ), blood urea nitrogen (normal range: $10.20-49.80 \mathrm{mg} / \mathrm{dl}$ ), serum UA (normal range: $3.40-7.20 \mathrm{mg} / \mathrm{dl}$ ), sodium, potassium, glucose, albumin, urinalysis and $24 \mathrm{~h}$ proteinuria. $\mathrm{sCr}$ was measured using a Jaffe alkaline picrate assay (Abbott Aeroset analyzer) (Wetzels et al., 2007). Serum UA was measured with an automatic analyzer (7700 series; Hitachi, Tokyo, Japan).

\section{Calculation of GFR}

GFR was calculated using the CKD-EPI equation, already validated in SSc patients (Gigante et al., 2012), expressed as a single equation: $\mathrm{GFR}=141 \times \min (\mathrm{sCr} / \mathrm{k}, 1) \alpha \times \max (\mathrm{sCr} / \mathrm{k}, 1)-1.209 \times 0.993$ Age $\times$ 1.018 (if female) $\times 1.159$ (if black), where $\mathrm{k}$ is 0.7 for females and 0.9 for males, $\alpha$ is -0.329 for females and -0.411 for males, min indicates the minimum of $\mathrm{sCr} / \mathrm{k}$ or 1 and max indicates the maximum of $\mathrm{sCr} / \mathrm{k}$ or 1 (Levey et al., 2009).

\section{Nailfold videocapillaroscopy (NVC)}

NVC was performed with a videocapillaroscope (Pinnacle Studio Version 8 ) equipped with a $500 \times$ optical probe. The nailfold of the

Table 1

\begin{tabular}{lc} 
SSc patients' epidemiological and clinical features. \\
\hline Sex (female/male) & $36 / 9$ \\
\hline Age, years & $45 \pm 13.8$ \\
Disease duration, years & $9 \pm 6$ \\
DAI & $3 \pm 2$ \\
DSS & $6 \pm 3$ \\
dcSSc/lcSSc & $28 / 17$ \\
SSc-specific autoantibodies n (\%) & $24(53)$ \\
Anti-topoisomerase I & $13(29)$ \\
Anticentromere & $8(18)$ \\
None & \\
Capillaroscopic pattern n (\%) & $13(28.9)$ \\
$\quad$ Early & $12(26.7)$ \\
Active & $20(44.4)$ \\
Late &
\end{tabular}

Disease Activity index (DAI); Disease Severity Scale (DSS); limited cutaneous SSc (lcSSc); diffuse cutaneous SSc (dcSSc). second, third, fourth and fifth fingers was examined in each patient. According to Cutolo et al. patterns identified within the "SSc pattern" include: early, active and late. The NVC is the best technique to evaluate microvascular damage in SSc patients (Cutolo et al., 2006).

\section{Clinical assessment}

Modified Rodnan total skin score (mRSS) was chosen as the most used method to assess skin induration in SSc. It is determined at a standardized location of 17 different sites of the body with a standardized pinching method and it is scored from 0 to 3 (Clements et al., 1995). Disease activity in SSc was measured using Disease Activity Index (DAI), which consists of 10 weighted variables: total skin score $>14$, scleroderma, digital necrosis, arthritis, total lung capacity $<80 \%$, erythrocyte sedimentation rate $(E S R)>30$, hypocomplementemia and change in cardiopulmonary, skin and vascular symptoms in the past month (Valentini et al., 2001). Disease severity was measured by Medsger Disease Severity Scale (DSS). The original scale assessed disease severity in 9 organs or systems, namely general health, peripheral vascular, skin, joint/tendon, muscle and gastrointestinal tract, lungs, heart and kidneys. Each organ/system is scored separately from 0 to 4 depending on whether there is no, mild, moderate, severe or end-stage involvement (Medsger et al., 1999).

\section{Doppler ultrasound}

SSc patients were placed for at least 15 min before the Doppler ultrasound examination in a temperature-controlled room at $22+0.4{ }^{\circ} \mathrm{C}$. The size of the left and right kidneys and the flow in the aorta and renal arteries were evaluated to detect a morphologic abnormality or renal artery stenosis. During the measurements, the patients were supine and held their breath. Doppler ultrasound examinations were performed by the same senior nephrologist blinded to the clinical features of the patient. Renal Doppler ultrasound was performed using a Toshiba Aplio Ultrasound System SSA-790 equipped with a convex 3.5-MHz probe. Renal Doppler flow was obtained in 3 different interlobar arteries of both kidneys (mesorenal, superior, and inferior pole), guided by color-flow mapping. The Doppler gate width was kept small, and the angle of insonation was maintained at $<60^{\circ}$. We used an anterior approach for detecting the renal artery origin and an oblique, lateral approach for the intermediate tract and intrarenal vessels. No aliasing was allowed in the interlobar arteries while the following parameters were measured: peak systolic velocity (PSV), end diastolic velocity (EDV), resistive index (RI), pulsatile index (PI), and systolic/diastolic ratio (S/D). RI was calculated as (peak systolic frequency shift- minimum diastolic frequency shift)/peak systolic frequency shift and the PI was calculated as (peak systolic frequency shift)/minimum diastolic frequency shift)/mean frequency shift. The PSV and EDV are expressed as $\mathrm{cm} / \mathrm{s}$. The mean value of 3 measurements of interlobar arteries from each kidney was calculated. For each patient, Doppler ultrasound parameters were calculated as the mean of measurements of both kidneys. While performing the renal Doppler examination, the operator evaluated the heart function. The Doppler examination was not performed in SSc patients with arrhythmia or tachycardia or bradycardia. Weighted kappa was used to evaluate the interrater reliability by the same observer. The kappa values for RI and S/D were 0.971 and 0.975 , respectively. The intrapatient coefficient of variation for RI and S/D measurement was $1.3 \%$ and $1.4 \%$, respectively. Calciumchannel blocker therapy was discontinued $72 \mathrm{~h}$ before the Doppler ultrasound (Rosato et al., 2012). The mean references value for normal RI in adults is determinate to be $0.60 \pm 0.10$, with 0.70 as the upper limit of normal (Parolini et al., 2009). Patients receiving iloprost therapy underwent Doppler examination the day before the next infusion. 


\section{Echocardiography}

Echocardiography was performed with an ultrasound Toshiba Ultrasound System (Tokyo, Japan) with a convex 2.5-3.5-MHz probe. All echocardiographic data were recorded according to the guidelines of the American Society of Echocardiography (ASE)[17]. PH was considered present when the systolic pulmonary artery pressure (PAPs) value $\geq 40 \mathrm{mmHg}$ (Mukerjee et al., 2004).

\section{Baseline pulmonary function tests}

Spirometric parameters of flows and volumes [ $\left(\mathrm{FEV}_{1}\right.$, forced expiratory volume in the 1 st second), FVC (Forced vital capacity), FEV $1 /$ FVC)] and single-breath carbon monoxide (CO) diffusing capacity (DLCO), corrected for hemoglobin concentration, were recorded with a Quark PFT 2 spirometer (Cosmed) and expressed according to the standards recommended by the American/European Respiratory Society. All spirometric parameters are expressed as percentage of predicted (Abboud and Sansores, 1996).

\section{Statistical analysis}

The results were expressed as mean and standard deviation (SD) or median and range, as appropriate. Commercial software (SPSS version 22.0) was used for the statistical analysis. The coefficient of skewness and the coefficient of kurtosis were used to evaluate the normal distribution of data. Multiple regression analysis was applied to evaluate the relationship between serum UA and the demographic and clinical features [age, disease duration (first symptom after RP), mRSS, DAI, DSS] and internal organ damage indices (creatinine, CKD-EPI, PAPs, PI, RI, S/D, DLCO, FVC). Pearson product-moment correlation coefficient (r) was used to test for an association between numerical variables. Group comparisons were made by Student's unpaired 2-tailed t-test or the Kruskal-Wallis test, as appropriate. P-values $<0.05$ were considered significant. A receiver operating characteristic (ROC) curve analysis was performed to analyze the prognostic accuracy of serum UA.

\section{Results}

Table 2 showed the mean values of serum UA, sCr, CKD-EPI, PAPs, Doppler ultrasound indices and pulmonary function tests. In the multiple regression analysis the serum UA showed a significant positive correlation with $\mathrm{sCr}(\mathrm{r}=0.33, \mathrm{p}<0.0001)$ and PAPs $(\mathrm{r}=0.38, \mathrm{p}<0,01)$ and negative correlation with CKD-EPI $(r=-0.35, p<0.01)$ (Fig. 1). No significant correlation ( $\mathrm{p}>0.05$ ) was observed between serum UA and age, DAI, DSS, mRSS, PI, RI, S/D, DLCO, FVC.

\begin{tabular}{|c|c|}
\hline Serum UA (mg/dl) & $4.24 \pm 1.23$ \\
\hline $\mathrm{sCr}(\mathrm{mg} / \mathrm{dl})$ & $0.75 \pm 0.16$ \\
\hline CKD-EPI (mil/min) & $101 \pm 18.9$ \\
\hline Proteinuria $(\mathrm{g} / 24 \mathrm{~h})$ & $0.13 \pm 0.04$ \\
\hline PI & $1.26 \pm 0.29$ \\
\hline RI & $0.64 / 0.06$ \\
\hline$S / D$ & $3.02 \pm 0.88$ \\
\hline Systolic blood pressure $(\mathrm{mmHg})$ & $112 \pm 9$ \\
\hline Diastolic blood pressure (mmHg) & $71 \pm 6$ \\
\hline PAPs (mmHg) & $28 \pm 5.4$ \\
\hline DLCO (\% of predicted) & $70 \pm 16$ \\
\hline FVC (\% of predicted) & $92 \pm 19$ \\
\hline BMI & $23 \pm 3.2$ \\
\hline
\end{tabular}

Uric acid (UA); serum creatinine (sCr); Chronic Kidney Disease Epidemiology Collaboration (CKD-EPI); systolic pulmonary artery pressure (PAPs); pulsatile index (PI); resistive index (RI); systolic/diastolic ratio (S/D); carbon monoxide diffusing capacity (DLCO); forced vitality capacity (FVC); Body mass index (BMI).
The mean value of serum UA was significantly $(\mathrm{p}<0.0001)$ different in three capillaroscopic groups: early $3.23 \pm 0.70$, active $3.86 \pm 1.12$ and late $5.14 \pm 0.91$ (Fig. 1). No significant ( $p>0.05$ ) difference of serum UA was observed in SSc patients with dcSSc $(4.3 \pm 1.3)$ or $\operatorname{lcSSc}(4.1 \pm 1.1)$.

Using a cut-off value of $4.7 \mathrm{mg} / \mathrm{dl}$ we grouped the SSc patients in two groups: patients with low-normal value of serum $U A(\leq 4.7 \mathrm{mg} / \mathrm{dl})$ and with high-normal serum UA ( $>4.7 \mathrm{mg} / \mathrm{dl})$. The mean value of serum UA in the first group $(\mathrm{n}=24)$ is $3.27 \pm 0.70(\mathrm{n}=24)$ and in the second group it is $5.36 \pm 0.55(n=21)$. The mean PAPs value was higher $(\mathrm{p}<0.01)$ SSc patients with high-normal serum UA than in those with low-normal serum UA ( $30 \pm 4 \mathrm{mmHg}$ vs $26 \pm 6 \mathrm{mmHg}$ ). The mean value of Doppler indices of intrarenal stiffness are significantly different $(\mathrm{p}<0.05)$ in SSc patients with low-normal serum UA or high-normal serum UA: PI was $1.13 \pm 0.22$ vs $1.40 \pm 0.30$, RI $0.62 \pm 0.05$ vs $0.66 \pm 0.07, \mathrm{~S} / \mathrm{D} 2.67 \pm 0.47$ vs $3.42 \pm 1.06$ (Table 2, Fig. 2 ). The ROC curves (AUC,\% and 95\% CI) demonstrated a good accuracy of serum UA for PAPs 73.7 (59.7-88.0, p $<0.01$ ), PI 75.7 (61.3-90.1, p $<0.01)$, RI 71.3 (56.6-81.6, $\mathrm{p}<0.05$ ) and S/D 71.9 (56.6-87.6, $\mathrm{p}<0.01)$. No significant difference of clinical variables was observed in the two groups of patients (Table 3).

\section{Discussion}

In our study serum UA shows a positive correlation with $\mathrm{sCr}$ and intrarenal arterial stiffness, conversely serum UA showed a negative correlation with CKD-EPI. UA is the final oxidation product of purine metabolism and it is excreted primarily by the kidney. The hyperuricemia frequently precedes the development of CKD. Various pathogenetic mechanisms (obesity, metabolic syndrome and arterial hypertension) might be involved in the pathogenesis of hyperuricemia (Johnson et al., 2013).

In our study the serum UA showed a linear positive correlation with PAPs. Several studies have demonstrated that increased serum UA concentration in patients with PAH correlates with a worse prognosis (Bendayan et al., 2003). Serum UA represents a prognostic predictor in PAH with connective tissue disease. The authors say that cytokine production, inflammation and endothelial dysfunction, which are considered to be among the primary events during the progression of the scleroderma-induced vasculopathy and fibrosis, can cause an increase in serum UA concentrations (Njaman et al., 2007). Then, Dimitroulas et al. have demonstrated that serum UA increased in proportion to the functional capacity in SSc patients with PAH (Dimitroulas et al., 2011). In the DETECT study serum UA represents one of the six nonechocardiographic variables to evaluate individual risk of PAH development in SSc patients. Hyperuricemia is considered a biomarker of renal hemodynamic impairment (Susic et al., 2015). In patients with essential hypertension, the serum UA concentration correlated inversely with renal blood and directly with renal vascular and total resistance. Hyperuricemia in patients with essential hypertension most likely reflects early renal vascular involvement, especially, nephrosclerosis (Messerli et al., 1980).

Our studies demonstrated that intrarenal vascular damage represents the first manifestation of renal damage in SSc patients. Intrarenal arterial stiffness, evaluated by Doppler indices, is higher in SSc patients than healthy controls. Doppler indices of intrarenal arterial stiffness show a negative correlation with eGFR and they progressively increase with severity of capillaroscopic damage (Gigante et al., 2015; Gigante et al., 2014; Rosato et al., 2013; Rosato et al., 2012).

Until today there are no studies that have shown an association between microvascular damage of skin and increased serum UA. Many of the severe internal organ complications of SSc are vascular (PAH, scleroderma renal crisis and reduction of GFR with increased of intrarenal arterial stiffness). Structural vascular damage occurs in many vascular districts and it contributes to pulmonary, renal, cardiac and gastrointestinal complications. In laboratory animals, hyperuricemia was also able 

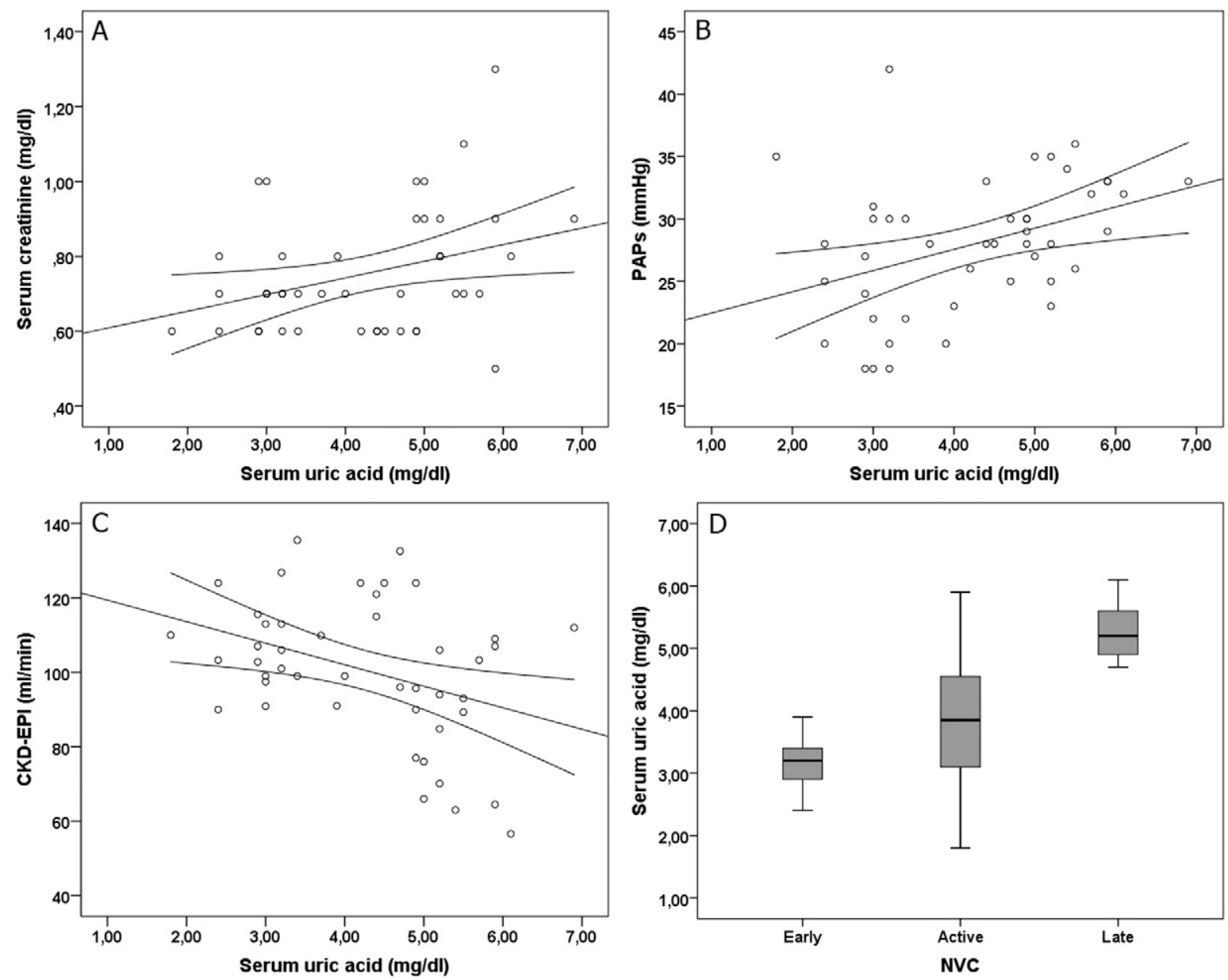

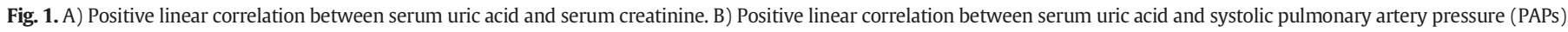

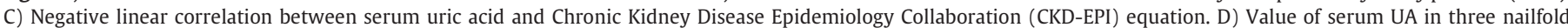
videocapillaroscopy (NVC) groups.

to induce oxidative stress and endothelial dysfunction, resulting in the development of both systemic and glomerular hypertension in association with elevated renal vascular resistance and reduced renal blood flow (Sanchez-Lozada et al., 2008; Sanchez-Lozada et al., 2005). Although this is the first report on the association between endothelial damage and serum UA in SSc patients, the important limitations of this study are represented by a limited number of patients, the absence of validation cohort and weak correlations. The serum UA concentration shows a good correlation with the capillaroscopic damage, with the PAPs values and estimated glomerular filtration rate. The division of the SSc population in the two cut-off was weakly significant and it needs further study to assess its usefulness.
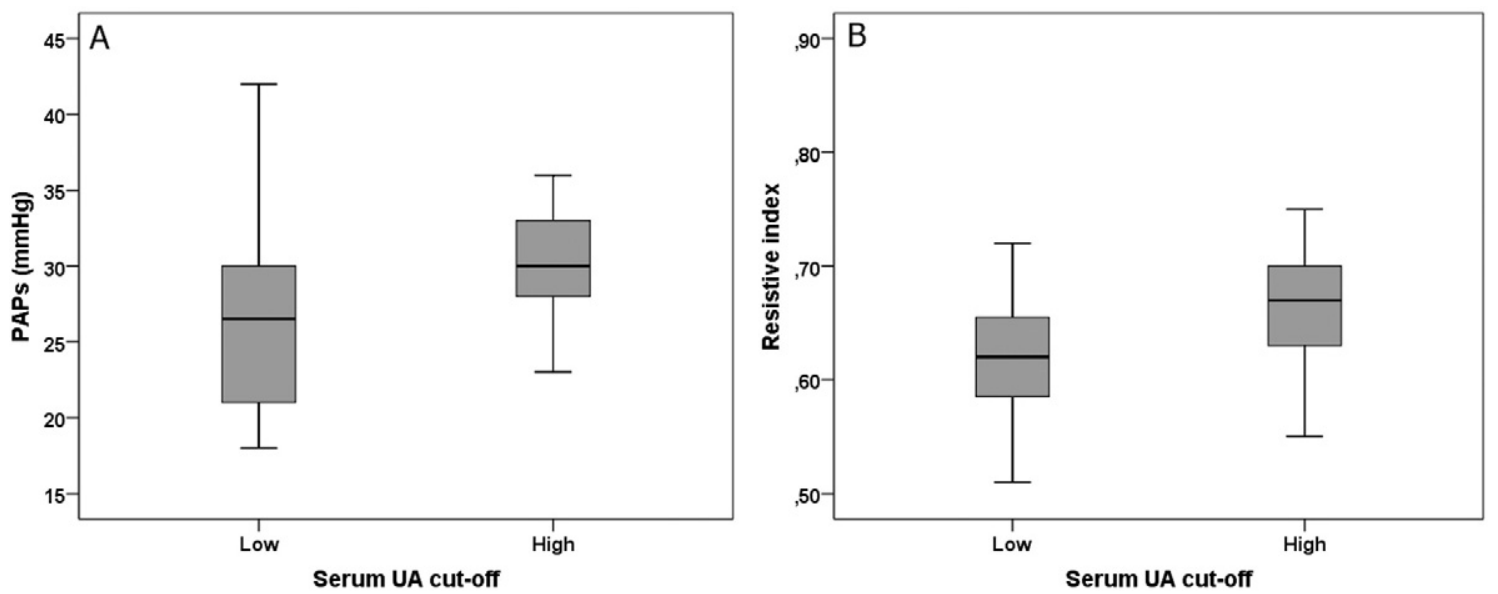

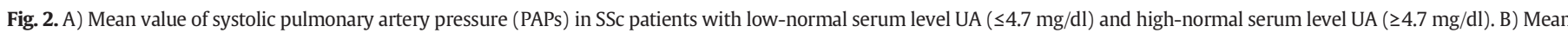
value of resistive index in patients with low normal serum level UA $(\leq 4.7 \mathrm{mg} / \mathrm{dl})$ and high normal serum level UA $(\geq 4.7 \mathrm{mg} / \mathrm{dl})$. 
Table 3

The mean value of PAPs, Doppler ultrasound indices, pulmonary function tests and clinical variables of disease in SSc patients with normal and high serum UA.

\begin{tabular}{lccc}
\hline & $\begin{array}{l}\text { Low normal } \\
\text { serum UA } \\
(\mathrm{n}=24)\end{array}$ & $\begin{array}{l}\text { High normal } \\
\text { serum UA } \\
(\mathrm{n}=21)\end{array}$ & p value \\
\hline PAPs (mmHg) & $26 \pm 6$ & $30 \pm 4$ & $<0.01$ \\
PI & $1.13 \pm 0.22$ & $1.40 \pm 0.30$ & $<0.05$ \\
RI & $0.62 \pm 0.05$ & $0.66 \pm 0.07$ & $<0.05$ \\
S/D ratio & $2.67 \pm 0.47$ & $3.42 \pm 1.06$ & $<0.05$ \\
DLCO (\% predicted) & $67 \pm 19$ & $72 \pm 14$ & $>0.05$ \\
FVC (\% predicted) & $90 \pm 20$ & $94 \pm 18$ & $>0.05$ \\
mRSS & $13 \pm 6$ & $13 \pm 6$ & $>0.05$ \\
DAI & $3 \pm 2$ & $3 \pm 2$ & $>0.05$ \\
DSS & $6 \pm 3$ & $6 \pm 4$ & $>0.05$ \\
\hline
\end{tabular}

Systolic pulmonary artery pressure (PAPs); pulsatile index (PI); resistive index (RI); systolic/diastolic ratio (S/D); carbon monoxide diffusing capacity (DLCO); forced vitality capacity (FVC); modified Rodnan skin score (mRSS); Disease Activity index (DAI); Disease Severity Scale (DSS).

In conclusion serum UA concentration is higher in patients with high microvascular damage than in patients with low microvascular damage. These preliminary data must be confirmed in large prospective studies.

\section{Conflicts of interest}

None.

\section{References}

Abboud, R.T., Sansores, R., 1996. ATS recommendations for DLCO. Am. J. Respir. Crit. Care Med. 154, 263.

Bendayan, D., et al., 2003. Hyperuricemia as a prognostic factor in pulmonary arterial hypertension. Respir. Med. 97, 130-133.

Clements, P., et al., 1995. Inter- and intraobserver variability of the total thickness score (modified Rodnan TSS) in systemic sclerosis. J. Rheumatol. 22, 1281-1285.

Coghlan, J.G., et al., 2013. Evidence-based detection of pulmonary arterial hypertension in systemic sclerosis: the DETECT study. Ann. Rheum. Dis. 73, 1340-1349.

Cutolo, M., et al., 2006. Nailfold capillaroscopy is useful for the diagnosis and follow-up of autoimmune rheumatic diseases. A future tool for the analysis of microvascular heart involvement? Rheumatology 45 (Suppl. 4), 43-46.

Dimitroulas, T., et al., 2011. Significance of serum uric acid in pulmonary hypertension due to systemic sclerosis: a pilot study. Rheumatol. Int. 31, 263-267.

Gigante, A., et al., 2012. Evaluation of chronic kidney disease epidemiology collaboration equation to estimate glomerular filtration rate in scleroderma patients. Rheumatology (Oxford) 51, 1426-1431.
Gigante, A., et al., 2014. Autonomic dysfunction in patients with systemic sclerosis: correlation with intrarenal arterial stiffness. Int. J. Cardiol. 177, 578-802.

Gigante, A., et al., 2015. Correlation between intrarenal arterial stiffness and exercise tolerance in systemic sclerosis patients without renal and cardiopulmonary impairment: the role of the microvascular damage. Int. J. Cardiol. 185, 122-124.

Hayden, M.R., Tyagi, S.C., 2004. Uric acid: a new look at an old risk marker for cardiovascular disease, metabolic syndrome, and type 2 diabetes mellitus: the urate redox shuttle. Nutr. Metab. (Lond.) 1, 10.

Johnson, R.J., et al., 2013. Uric acid and chronic kidney disease: which is chasing which? Nephrol. Dial. Transplant. 28, 2221-2228.

LeRoy, E.C., et al., 1998. Scleroderma (systemic sclerosis): classification, subsets and pathogenesis. J. Rheumatol. 15, 202-205.

Levey, A.S., et al., 2009. CKD-EPI (Chronic Kidney Disease Epidemiology Collaboration): a new equation to estimate glomerular filtration rate. Ann. Intern. Med. 150, 604-612.

Matucci-Cerinic, M., et al., 2013. Systemic sclerosis (scleroderma, SSc) is a vascular disease. Arthritis Rheum. 65, 1953-1962.

Medsger Jr., T.A., et al., 1999. A disease severity scale for systemic sclerosis: development and testing. J. Rheumatol. 26, 2159-2167.

Messerli, F.H., et al., 1980. Serum uric acid in essential hypertension: an indicator of renal vascular involvement. Ann. Intern. Med. 93, 817-821.

Mukerjee, D., et al., 2004. Echocardiography and pulmonary function as screening tests for pulmonary arterial hypertension in systemic sclerosis. Rheumatology 43, 461-466.

Njaman, W., et al., 2007. Serum uric acid as a prognostic predictor in pulmonary arterial hypertension with connective tissue disease. Int. Heart J. 48, 523-532.

Parolini, C., et al., 2009. Renal resistive index and long-term outcome in chronic nephropathies. Radiology 252, 888-896.

Rosato, E., et al., 2012. Intrarenal hemodynamic parameters correlate with glomerular filtration rate and digital microvascular damage in patients with systemic sclerosis. Semin. Arthritis Rheum. 41, 815-821.

Rosato, E., et al., 2013. Doppler indices of intrarenal arterial stiffness are useful in monitoring scleroderma renal crisis. Scand. J. Rheumatol. 42, 80-81.

Sanchez-Lozada, L.G., et al., 2005. Mild hyperuricemia induces vasoconstriction and maintains glomerular hypertension in normal and remnant kidney rats. Kidney Int. 67, 237-247.

Sanchez-Lozada, L.G., et al., 2008. Role of oxidative stress in the renal abnormalities induced by experimental hyperuricemia. Am. J. Phys. 295, 1134-1141.

Shanmugam, V.K., Steen, V.D., 2012. Renal disease in scleroderma: an update on evaluation, risk stratification, pathogenesis and management. Curr. Opin. Rheumatol. 24, 669-676.

Sowers, J.R., 2013. Diabetes mellitus and vascular disease. Hypertension 61, 943-947.

Susic, D., et al., 2015. A biomarker of renal hemodynamic impairment. Cardiorenal Med. 5, 175-182.

Valentini, G., et al., 2001. European multicentre study to define disease activity criteria for systemic sclerosis. II. Identification of disease activity variables and development of preliminary activity indexes. Ann. Rheum. Dis. 60, 592-598.

van den Hoogen, F., et al., 2013. Classification criteria for systemic sclerosis: an American College of Rheumatology/European League Against Rheumatism collaborative initiative. Arthritis Rheum. 65, 2737-2747.

Wetzels, J.F., et al., 2007. Age- and gender specific reference values of estimated GFR in Caucasians: the Nijmegen Biomedical Study. Kidney Int. 72, 632-637. 